\title{
News from PR China
}

\section{The sixteenth China Patent Award}

China Patent Award is a government award which was set up by the State Intellectual Property Office (SIPO) and the World Intellectual Property Organization (WIPO) to honor the patented inventions and designs, aiming to thoroughly implement intellectual property strategy and innovation-driven development strategy, to promote the use of intellectual property rights, and to accelerate economic transformation and upgrading. The criteria for the award not only emphasize the patent level of projects, the height of innovation, and the promotion effects on technology progress, but also lay stress on patent transformation process in market and significant impact on domestic innovation achievements, especially the comprehensive benefits of patents. China Patent Award was set up in 1989. From the eleventh session in 2009, the award cycle changed from two years to one year.

In the sixteenth China Patent Award, twenty patents were chosen as candidates of Chinese Patent Gold Awards, such as "A shift register unit, a gate driver for display and liquid crystal display". Five candidates of Chinese Appearance Design Gold Awards were voted, such as "An electronic device with display function". The awards committee picked 408 patents as candidates of China Patent Excellence Award, such as "A method for the hydraulic optimization of centrifugal pump based on CFD in multi conditions" and 64 patents as candidates of China Appearance Design Excellence Awards, such as "A LED lighting lamp". Beijing Intellectual Property Office and other seven organizations got the Best Organization Awards. Twenty organizations, such as Liaoning Intellectual Property Office, were awarded Excellence Organization Award. The award of award-winning inventors (designers) would be recorded in the personal files as an important basis for evaluation, promotion, and appointment. Organization where the inventors (designers) work for or the competent department at a higher level should give appropriate incentives to the inventors (designers).

http://www.sipo.gov.cn/tz/pingxuan/201411/t20141125_ 1037630.html

Ying-Qi Xu

Chengdu Documentation and Information Center, Chinese Academy of Sciences

\section{The eighth China Patent Week}

On November 17, 2014, the eighth China Patent Week was launched. The theme of this patent week was "focusing on the needs of enterprises, serving innovation and development".

Focusing on the key work such as the promotion plan of patent strategy, the pilot project of patent navigation, popularizing the national standard "Rules of Enterprise Intellectual Property Management" and promoting the development of micro businesses, this patent week displayed the full-range work involving patent creation, application, protection, management and service to create a good atmosphere for serving enterprise innovation and promoting the social innovation and development. This patent week pay attention to the enterprises' needs. Based on the practical needs of enterprises, the organizers gathered all kinds of resources concerning intellectual property services to carry out joint activities of intellectual property services for enterprises, providing high targeted quality service for the innovation of enterprises, in order to solve the enterprises' problems of intellectual property. The activities of this patent week had four features, including enhancing the enterprises' management of intellectual property, promoting competition advantage of intellectual property, cultivating enterprises' ability of strengthening intellectual property application, and strengthening the intellectual property service for small and medium sized enterprises.

This session of China Patent Week was launched on China Intellectual Property Network. The sections of video broadcast, program of activities, real-time live broadcast, etc. were set up to pass the real-time event information to web users by texts, pictures, video and other forms. Meanwhile, the organizers of China Intellectual Property Network used mobile SNS application to widely spread the latest activities information to mobile phone users. This patent week made full use of network communication means. Large capacity and a wide range of reports focused on this patent week to form an overall effect and further build the brand effect and lasting effect of China Patent Week.

Some local events are as follows.

Shanghai Intellectual Property Office held on symposiums on improving companies' ability to use intellectual property and serving small and micro enterprises' intellectual property.

The first Jiangxi university patent trade fair was held by Jiangxi Department of Science and Technology, Department of Education, Industry and Information Committee, and Intellectual Property Office.

Sichuan Intellectual Property Office held an opening ceremony of 8th China Patent Week activities in Sichuan and a symposium on using IP to boost park industries.

Guangxi Intellectual Property Office conducted propaganda of intellectual property knowledge through community LED screen and Guangxi TV weather forecast section.

An opening ceremony of 8th China Patent Week activities in Fujian and a biotechnology patent matchmaking symposium were held by Fujian Intellectual Property Office, Fuzhou University, Fujian Normal University, Fujian Agriculture And Forestry University, Fujian Medical University and Fujian Academy of Agricultural Sciences.

A series of activities were carried out in Guangdong Province, including the opening activities of Guangdong intellectual property innovation experimental area, organizing outstanding patent projects of Guangdong Province to participate in the Sixteenth 
Shenzhen Hi-Tech Fair, the development forum of Guangdong province's patent agencies, the tour seminar of American intellectual property system, the training on enterprise intellectual property management standards, the seminar of patent value analysis for promoting patent mortgage financing, and 2014 China (Guangdong) matchmaking symposium of intellectual property financing projects.

http://www.sipo.gov.cn/zscqgz/2014/201411/t20141119_ 1034190.html

http://www.sipo.gov.cn/ztzl/ndcs/zgzlz/dbjzgzlz/

http://www.sipo.gov.cn/zscqgz/2014/201411/t20141114_ 1031718.html

http://www.sipo.gov.cn/zscqgz/2014/201411/t20141113_ 1031110.html

Ying-Qi Xu

Chengdu Documentation and Information Center, Chinese Academy of Sciences

\section{Five-year targets of local intellectual property strategies have been nearly implemented}

On November 25, 2014, the State Intellectual Property Office (SIPO) released the implementation status of Chinese local intellectual property strategies, and published "Instruction Manual of Chinese Local Intellectual Property Strategy Implementation" ("Manual" for short) at the same time. Twenty-five provinces accounting for $83 \%$ of the total provinces have completed local intellectual property strategies' targets. Five-year targets of local intellectual property strategies have been nearly implemented with the joint efforts of national intellectual property system.

In 2013, thirty provinces (autonomous regions, municipalities) were chosen as independent samples to be evaluated in the national evaluation of local intellectual property strategies. Overall evaluation results indicated that there was a distinctly local characteristic distribution of intellectual property development with close relationship to the local economic development. Intellectual property development nearly adapts to local economic development. Eight provinces (municipalities) with the highest intellectual property development level, including Beijing, Tianjin, Liaoning, Shandong, Jiangsu, Shanghai, Zhejiang, Guangdong, locate in Bohai Economic Rim or southeast coastal area with the best economic vigor. the intellectual property development levels of eight provinces which all but Fujian locate in central or western China. are higher. Intellectual property development levels of 7 provinces, most of which locate in northeastern and southwestern China. are medium. There are seven provinces which mainly locate in northwestern underdeveloped regions with low intellectual property development level. Subentry evaluation results indicated that $80 \%, 77 \%, 87 \%, 87 \%$ and $77 \%$ provinces were qualified on intellectual property innovation, application, protection, management and service, respectively.

SIPO compiled "Manual" to promote implementation of local intellectual property strategy and construction of powerful intellectual property provinces or cities. The "Manual" consisted of five parts. First, it introduces the promoting system of Chinese local intellectual property strategy implementation. Then, following three parts separately present position, application system and key work of provinces', cities', and counties' intellectual property strategy implementation. The last part is based on "National Intellectual Property Strategy Outline", describes the key content in local intellectual property strategies, through the conclusion of six-year results and experience concerning local intellectual property strategy implementation and promotion, including improving output of intellectual property, promoting application of intellectual property, strengthening protection of intellectual property, improving service of intellectual property and promoting construction of intellectual property regions, to provide reference to the local intellectual property strategy implementation.

http://www.sipo.gov.cn/zscqgz/2014/201411/t20141126_ 1037869.html

Ying-Qi Xu

Chengdu Documentation and Information Center, Chinese Academy of Sciences

\section{Implementation of automatic electronic exchange of priority documents between SIPO and USPTO}

The State Intellectual Property Office of the People's Republic of China was pleased to announce that from October 8, 2014, it would implement automatic electronic exchange of priority documents with the United States Patent and Trademark Office (USPTO).

This electronic exchange service will benefit patent applicants at SIPO and USPTO by reducing the administrative costs of ordering certified copies of priority documents, and will provide priority documents to the office of second filing in time.

This service is free of charge and not subject to any formalities on the part of the applicant. The U.S. patent application, the P.R.C.'s national patent or utility model application claiming priority from a first filing in the other office must have been filed on or after October 8, 2014.

Didi Liu

National Museum of China

http://www.sipo.gov.cn/zscqgz/2014/201409/t20140926_ 1015280.html

\section{The eighth International Exhibition of Inventions}

The eighth International Exhibition of Inventions opened at the Huaqiao International Expo Center in Kunshan, on November 19, 2014. The fair was co-hosted by the China Association of Inventions (CAI) and the International Federation of Inventors' Associations (IFIA).

The fair's theme was:"meeting at the beautiful Kunshan, let the dream of invention flying". There were more than 1000 booths in the 20,000-square-meter exhibition area. The fair attracted participants from more than 30 countries and regions, including USA, Germany, and South Korea. More than 3000 invention projects were displayed during the fair which would close on November 22.

Dr. Andras Vedres, president of International Federation of Inventors' Associations, said at the opening ceremony that there would be no inventions without inventors, no innovation without inventions, and no global prosperity without innovation. Innovation is the most powerful driving force for the economic growth. China ranked first in the world with its 561,000 new inventions in 2013, showing a strong momentum in innovationoriented economy.

Compared with the previous fairs, the eighth International Exhibition of Inventions showed more new inventions and technologies. The event featured a "maker area" where more than 100 creators from home and abroad would share their ideas and achievements such as 3D print and so on. There was also a special zone known as TRIZ in where the inventors show the inventions created by the method of TRIZ.

In addition to the inventions show, a string of forums regarding youth innovation, intellectual property, TRIZ training will also be held. In addition, IFIA will host a conference concurrently to select its new chairman. 
The exhibition made the enterprises and inventors together to build up an internationally well-known brand for an invention fair, where people can share their expertise and trade in patented technologies.

Didi Liu

National Museum of China

http://www.sipo.gov.cn/tz/hy/201404/t20140422_936843.html

http://www.inventionexpo.cn/about/

http://www.js.xinhuanet.com/2014-11/20/c_1113332521_2.htm
Ying-Qi Xu ${ }^{\mathrm{a}}$, Didi Liu ${ }^{\mathrm{b}}$, Zhiping Yang,* ${ }^{a}$ Chengdu Documentation and Information Center, Chinese Academy of Sciences, China

${ }^{\mathrm{b}}$ National Museum of China, China

${ }^{*}$ Corresponding author. Tel.: +86 13018265002. E-mail addresses: yangzp@yahoo.com, yangzp@clas.ac.cn (Z. Yang).

Available online 11 February 2015 